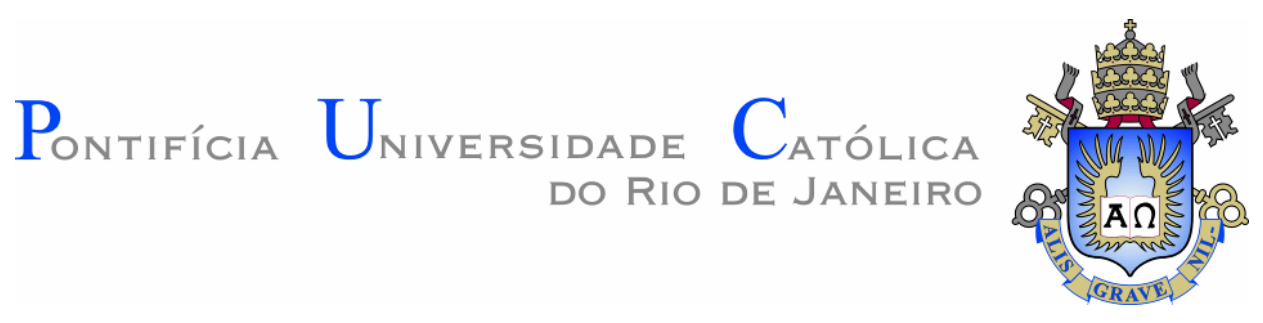

Lili Edith Daza Durand

\title{
Classificação de Hematitas em Minério de Ferro: Otimização de Aquisição e Processamento de Imagens
}

Dissertação de Mestrado

Dissertação apresentada como requisito parcial para obtenção do título de Mestre pelo Programa de Pós-Graduação em Engenharia de Materiais e de Processos Químicos e Metalúrgicos do Departamento de Engenharia Química e de Materiais da PUC-Rio.

Orientador: Prof. Sidnei Paciornik

Co-orientador: Dr. Julio César Álvarez Iglesias

Rio de Janeiro

Agosto de 2015 


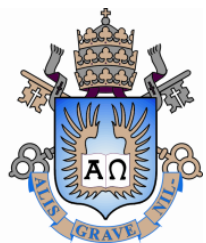

Lili Edith Daza Durand

\section{Classificação de Hematitas em Minério de Ferro: Otimização de Aquisição e Processamento de Imagens \\ Dissertação apresentada como requisito parcial para obtenção do título de Mestre pelo Programa de Pós-Graduação em Engenharia de Materiais e de Processos Químicos e Metalúrgicos do Departamento de Engenharia Química e de Materiais do Centro Técnico Científico da PUC-Rio. Aprovada pela Comissão Examinadora abaixo assinada.}

Prof. Sidnei Paciornik Orientador e Presidente Departamento de Engenharia Química e de Materiais - PUC Rio

Dr. Julio César Álvarez Iglesias Co - Orientador Departamento de Engenharia Química e de Materiais - PUC Rio

Dr. Marcos Henrique de Pinho Maurício Departamento de Engenharia Química e de Materiais - PUC Rio

Dr. Otávio da Fonseca Martins Gomes Centro de Tecnologia Mineral - CETEM

Prof. José Eugenio Leal Coordenador Setorial do Centro Técnico Científico - PUC-Rio 
Todos os direitos reservados. É proibida a reprodução total ou parcial do trabalho sem autorização da universidade, do autor e do orientador.

\section{Lili Edith Daza Durand}

Graduou-se em Engenharia Química pela Universidad Nacional de Ingenieria do Perú. Ingressou no curso de mestrado em Engenharia de Materiais no ano 2013.

Ficha Catalográfica

Daza Durand, Lili Edith

Classificação de Hematitas em Minério de Ferro: Otimização de Aquisição e Processamento de Imagens / Lili Edith Daza Durand ; orientador: Sidnei Paciornik ; co-orientador: Julio César Álvarez Iglesias. - 2015.

94 f. : il. (color.) ; $30 \mathrm{~cm}$

Dissertação (mestrado) - Pontifícia Universidade Católica do Rio de Janeiro, Departamento de Engenharia Química e de Materiais, 2015.

Inclui bibliografia

1. Engenharia Química - Teses. 2. Engenharia de Materiais - Teses. 2. Minério de Ferro. 3. Hematita. 4. Classificação Automática. 5. Análise de Imagens. I. Paciornik, Sidnei. II. Iglesias, Julio César Álvarez. III. Pontifícia Universidade Católica do Rio de Janeiro. Departamento de Engenharia Química e de Materiais. IV. Título. 
A Deus, por tudo o que sou.

A Juliana minha mãe pela dedicação, compreensão, paciência e amor que fizeram possível minha realização professional e pessoal. 


\title{
Agradecimentos
}

A Deus pela oportunidade de sair de meu pais para poder crescer como professional e como ser humano.

A CAPES Coordenação de Aperfeiçoamento de Pessoal de Nível Superior pela ajuda financeira.

A Juliana e Francisco, meus pais, a Gladys y Maritza minhas irmãs, por apoiarme e confiar em mim ao largo desta travessia; e que apesar da distância sempre senti o amor de cada um deles.

A meu orientador Sidnei Paciornik pelos conhecimentos e oportunidades brindadas no processo de formação como mestre, pela confiança que deposito em mim e pôr as palavras de alento que me impulsavam a fazer melhor as coisas.

\begin{abstract}
A meu co-orientador Julio César Álvarez Iglesias pelo ensinamento, compromisso, a paciência e pela ajuda brindada ao longe do desenvolvimento da minha teses.
\end{abstract}

Ao professor Marcos Henrique pelo tempo, a paciência e o conhecimento transmitido durante os momentos de dúvida. A Karen Augusto Soares pelo tempo fornecido e por estar sempre disposta a ajudar-me com alguma dúvida.

A meu noivo Roger Gago Tolentino, por seu amor, compreensão, pelas palavras de alento em momentos difíceis e por ser parte deste logro.

A minha amiga Lilia Olaya Luengas, pela agradável companhia durante todo o Mestrado e pelas experiências compartilhadas.

E a cada pessoa com a que compartilhei momentos, risos e palavras. 


\section{Resumo}

Daza Durand, Lili Edith; Paciornik, Sidnei; Álvarez Iglesias, Julio César. Classificação de Hematitas em Minério de Ferro: Otimização de Aquisição e Processamento de Imagens. Rio de Janeiro, 2015. 94p. Dissertação de Mestrado - Departamento de Engenharia Química e de Materiais, Pontifícia Universidade Católica do Rio de Janeiro.

O minério de ferro é um material policristalino oriundo de processos naturais complexos. Os minerais mais comuns que o compõem (hematita, magnetita, goethita, etc.) podem ser identificados no microscópio ótico de luz refletida, através de suas refletâncias distintas. A importância do estudo das hematitas, especificamente, surge porque os maiores depósitos de minério de ferro, no Brasil, são praticamente todos do tipo hematítico, com altos teores de ferro. A hematita é um mineral fortemente anisotrópico que apresenta pleocroísmo de reflexão. Isto faz com que o brilho na imagem mude com diferentes orientações dos cristais. Assim, quando se utiliza luz polarizada, o contraste entre os cristais aumenta o suficiente para diferenciá-los. Tradicionalmente, as hematitas são classificadas em "tipos texturais" identificados como hematita microcristalina (Mc), martita (Ma), e partículas policristalinas compactas (Co) formadas, por sua vez, de cristais dos tipos: granular $(\mathrm{Gr})$, lamelar (La), lobular (Lo). Em trabalhos anteriores foi desenvolvida uma rotina de classificação automática para os diferentes tipos de hematitas. Esta rotina utiliza como entrada duas imagens de uma mesma região, a primeira em campo claro (CC) e a segunda polarizada circularmente (CPOL). Neste trabalho foram implementadas modificações nas etapas de aquisição das imagens CPOL e no processamento de ruído, visando melhorar as etapas de classificação. Assim, a imagem CPOL, que apresenta problemas característicos de iluminação, passou a ser capturada utilizando o recurso de sub-quadros (subframe), o que elimina a necessidade de correção de fundo, melhorando a qualidade dos mosaicos de imagens capturados. Em seguida, explorou-se recurso de saturação digital da câmera, de forma a melhorar, substancialmente, o contraste entre os cristais de hematita. Finalmente, testou-se o impacto do uso de um novo filtro de redução de ruído - Non-Local Means (MNL) - sobre a segmentação de cristais. Os resultados mostraram uma melhora substancial na identificação dos tipos texturais de hematita com relação aos métodos anteriores e também superiores à tradicional identificação visual por operador treinado. 


\section{Palavras-Chave}

Minério de Ferro; Hematita; Classificação Automática; Análise de Imagens. 


\section{Abstract}

Daza Durand, Lili Edith; Paciornik, Sidnei (Advisor); Álvarez Iglesias, Julio César (Co-Advisor). Classification of Hematites in Iron Ore: Optimization of Image Acquisition and Processing. Rio de Janeiro, 2015. 94p. Masters Dissertation - Departamento de Engenharia Química e de Materiais, Pontifícia Universidade Católica do Rio de Janeiro.

Iron ore is a polycrystalline material originated from complex natural processes. Its main composing minerals (hematite, magnetite, goethite, etc.) can be identified with the reflected light optical microscope through their distinctive reflectances. The relevance of studying hematite, specifically, originates from the fact that the largest Brazilian iron deposits are mostly of the hematitic type, with high iron content. Hematite is a strongly anisotropic mineral that presents reflectance pleocroism. Thus, different crystal orientations produce different brightness and, when using polarized light, the contrast between crystals is strong enough to allow their discrimination. Traditionally, hematites are classified in textural types identified as microcrystalline (Mc), martite (Ma) and compact polycristalline (Co), composed of granula ( $\mathrm{Gr}$ ), lamellar (La) and lobular (Lo) crystals. An automatic classification routine for hematite types was developed in previous works. This routine takes as input two images of the same region, one in Bright Field and the second in Circular Polarization (CPOL). In this work, modifications in the CPOL image acquisition and in noise filtering were implemented, in order to improve the classification step. Thus, the CPOL images, which present a characteristic background problem, were acquired employing the subframe method, what eliminates the need for background correction, improving the quality of image mosaics. Then, the digital saturation of the camera was optimized to improve substantially the contrast between hematite types. Finally, the impact of a new noise reduction filter - the Non-Local Means Filter - on crystal segmentation was evaluated. The results showed a substantial improvement in the identification of hematite textural types as compared to the previous method, and also superior to the traditional visual identification by an operator.

\section{Keywords}

Iron Ore; Hematite; Automatic Classification; Image Analysis. 


\section{Sumário}

1 Introdução

2 Objetivos

3 Revisão Bibliográfica

19

3.1. Minério de Ferro

19

3.2. Hematita

21

3.3. Microscopia 23

3.3.1. Microscopia Digital 23

3.3.2. Microscopia Ótica de Luz Refletida em Campo Claro 25

3.3.3. Luz Polarizada $\quad 25$

3.3.4. Polarização Circular 26

3.3.5. Aquisição Automática de Imagens 28

3.4. Processamento e Análise Digital de Imagens $\quad 29$

3.4.1. Registro de Imagem 30

3.4.2. Correção de Fundo 32

3.4.3. Filtragem de Ruído 33

3.4.4. Técnicas de segmentação $\quad 34$

3.4.4.1. Limiarização $\quad 34$

3.4.4.2. Segmentação por Textura $\quad 35$

3.4.4.3. Crescimento de Regiões $\quad 40$

3.4.5. Espaço de Medidas 42

3.4.6. Reconhecimento de Padrões e Classificação 45

$\begin{array}{ll}\text { 3.4.6.1. Classificação Supervisionada } & 47\end{array}$

$\begin{array}{ll}4 \text { Materiais e Métodos } & 48\end{array}$

4.1. Preparação de Amostras $\quad 48$

4.2. Aquisição de Imagens ao Microscópio Ótico 49

4.3. Desalinhamento 52

4.4. Segmentação $\quad 55$

4.4.1. Limiarização 55

4.4.2. Crescimento de Regiões 56

4.5. Classificação $\quad 60$

4.5.1. Classificação por Textura (Método Sintético) 60 
5 Resultados e Discussão 64

5.1. Otimização do Frame da Câmera 64

5.2. Análise Qualitativa das Diferentes Técnicas de Captura 65

5.2.1. Comparação de imagens Full Frame com Subframe 65

5.2.2. Comparação de Imagens Subframe com Subframe Saturadas 67

5.2.3. Comparação de Imagens Subframe saturadas com Subframe saturadas NLM 68

5.3. Analise Estatística do Método Sintético 69

5.4. Análise Qualitativa do Método Analítico 72

5.5. Análise Quantitativa dos Resultados 75

6 Conclusões e Trabalhos Futuros 79

6.1. Trabalhos Futuros 80

7 Referências Bibliográficas $\quad 81$

8 Anexo $\quad 85$

8.1. Resultados 85

8.1.1. Otimização da posição do subframe frame 85

8.1.2. Otimização do frame da Câmera 87

8.1.3. Análise Qualitativa do Método Analítico 90

8.1.4. Análise Quantitativa dos Resultados 92 


\section{Lista de Figuras}

Figura 3-1: Microscópio ótico do LMD da PUC-Rio..........................................24

Figura 3-2: Esquema da Luz Refletida em Campo Claro [22] .........................25

Figura 3-3: Esquema da formação da luz polarizada. (a) Imagem

LPOL com polarizador girado $-10^{\circ}$ e (b) Imagem LPOL com

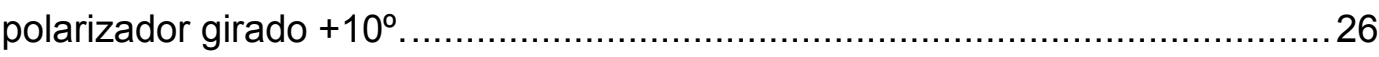

Figura 3-4: Esquema da formação da luz polarizada circular [22] ...................2 27

Figura 3-5: Técnica de captura do mosaico; (a) varredura espacial com

captura de imagens individuais; (b) concatenação das imagens e

criação do mosaico; (c) visão ampliada para exemplificar a

superposição dos campos verde e amarelo [20]............................................29

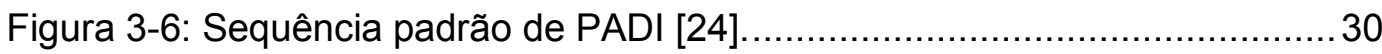

Figura 3-7: Possíveis transformações em registro de imagens [27]. .................. 31

Figura 3-8: Ilustração do processo de correção de iluminação. (a) Imagem

original, (b) Imagem corrigida.

Figura 3-9: Ilustração do efeito do filtro NLM. (a) Imagem original;

(b) Imagem depois da aplicação NLM 34

Figura 3-10: Representação gráfica do vetor d [24].

Figura 3-11: Exemplo de crescimento de regiões com t=3: (a) Imagem em tons de cinza; (b) Duas regiões (R1 e R2) separadas com $n=2$ sementes (em vermelho); (c) Três regiões (R1, R2, e R3) separadas com $n=3$ sementes (em vermelho) [17]

Figura 3-12 Conectividade entre os pixels: (a) Imagem original, onde o pixel circulado é o pixel analisado; (b) Conectividade 4, considerando que são dois objetos separados; (c) Conectividade 8, considerando que os pixels fazem parte de um mesmo objeto [6]

Figura 3-13: Classificação supervisionada utilizando os parâmetros característicos FFC e RA [17].

Figura 4-1: Propriedades da câmera CCD. 50

Figura 4-2: Ilustração de captura de imagens. (a) Com quadro

completo (full frame); (b) Com quadro parcial (subframe).

Figura 4-3: Imagens CC e CPOL, respectivamente, desalinhadas.

Para perceber melhor o desalinhamento parte das imagens $\mathrm{CC} \mathrm{e}$

CPOL foram ampliadas.

Figura 4-4: Imagens CC e CPOL, respectivamente, alinhadas. Para 
perceber melhor o alinhamento parte das imagens CC e CPOL

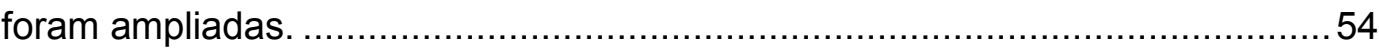

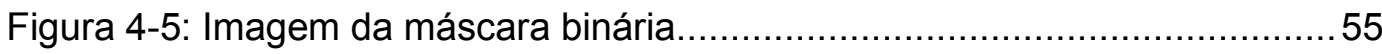

Figura 4-6: Interseção da Máscara binária (Figura 4-5) com CPOL

(Figura 4-4(b)) utilizando o operador lógico AND.........................................56

Figura 4-7: Imagem CPOL com ruído gaussiano. ..........................................5 57

Figura 4-8: Imagem resultante do Canny sobre a imagem com ruído. ..............57

Figura 4-9: Imagem resultante do divisor de águas. ......................................58

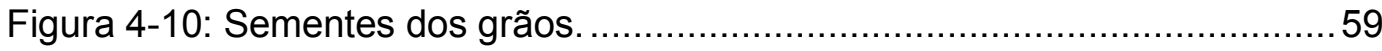

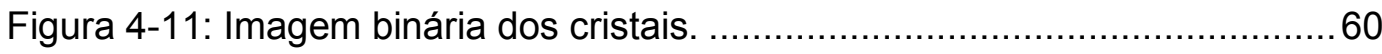

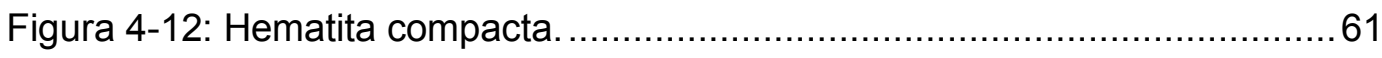

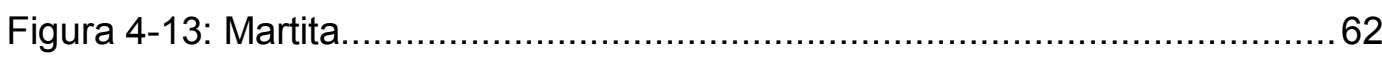

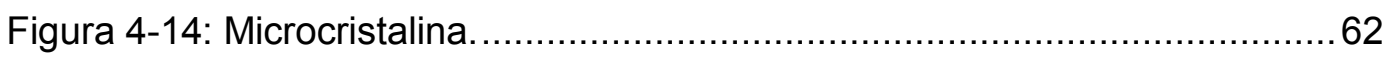

Figura 4-15: Cristais de hematita compacta classificados...............................63

Figura 5-1: Tipos de aquisição de imagens CPOL: (a) Mosaico 2x2 com

full frame; (b) Mosaico 28x21 subframe de 200x200.

Figura 5-2: Fração dos mosaicos originais da Figura 5-1 que

mostram a comparação entre as imagens: (a) CPOL full frame $2 \times 2$ com

gradiente de iluminação (b) CPOL full frame com gradiente de

iluminação corrigido; (c) CPOL subframe

Figura 5-3: Comparação entre as imagens: (a) CPOL subframe e;

(b) CPOL subframe saturada.

Figura 5-4: Comparação entre as imagens: (c) CPOL subframe saturada

e; (d) CPOL subframe saturadas NLM.

Figura 5-5: Representação da matriz de confusão para martita.

Figura 5-6: Exemplo de Identificação de cristais em função das

variações da imagem CPOL. (a) CPOL full frame; (b) CPOL sub frame;

(c) CPOL subframe saturada; (d) CPOL subframe saturadas NLM.

Figura 5-7: Comparação entre as frações de hematita compacta obtidas pelos métodos sintético e analítico para o Mosaico 1.

Figura 5-8: Imagens da Classificação de hematita. (a) Imagem CC;

(b) Imagem CPOL (c) Imagem full frame; (d) Imagem subframe;

(e) Imagem subframe saturada; (f) Imagem subframe saturada NLM.

Figura 8-1:Mosaico 5x5 subframe: (a) framestart não centrado;

(b) framestart centrado

Figura 8-2: Mosaico 6x4 subframe: (a) framestart não centrado;

(b) framestart centrado. 
Figura 8-3: Mosaico $2 \times 2$ com full frame .87

Figura 8-4: Mosaico 5x5 subframe de 1292x976......................................... 87

Figura 8-5: Mosaico 6x4 subframe de $1024 \times 1024$........................................ 88

Figura 8-6: Mosaico 11x8 subframe de 512x512....................................... 88

Figura 8-7: Mosaico 22x16 subframe de 256x256...................................... 89

Figura 8-8: Exemplo de Identificação de cristais em função das variações da imagem CPOL. (a) CPOL full frame; (b) CPOL subframe;

(c) CPOL subframe saturada; (d) CPOL subframe saturadas NLM. 90 Figura 8-9: Exemplo de Identificação de cristais em função das variações da imagem CPOL. (a) CPOL full frame; (b) CPOL subframe;

(c) CPOL subframe saturada; (d) CPOL subframe saturadas NLM. 91

Figura 8-10: Comparação entre as frações de hematita compacta obtidas pelos métodos sintético e analítico para o Mosaico 2. 92

Figura 8-11: Comparação entre as frações de hematita compacta obtidas pelos métodos sintético e analítico para o Mosaico 3. .93 


\section{Lista de Tabelas}

Tabela 3-1: Reservas mundiais de minério de ferro no ano 2013 [14] ..............21

Tabela 3-2: Tipos de hematita compacta [16] .............................................. 22

Tabela 3-3: Tipos de hematita não compacta [16] .......................................22

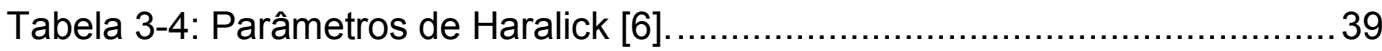

Tabela 5-1: Taxa de acerto da classificação nas imagens CPOL. ......................69

Tabela 5-2: Matriz de confusão para imagens full frame................................. 70

Tabela 5-3: Matriz de confusão para imagens subframe ................................. 70

Tabela 5-4: Matriz de confusão para imagens subframe saturadas. ..................71

Tabela 5-5: Matriz de confusão para imagens subframe saturadas NLM...........71

Tabela 5-6: Fração de área das hematitas (\%), para as 4 condições

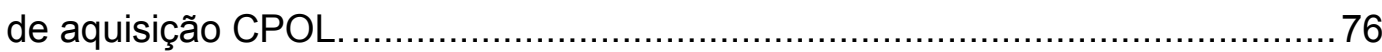

Tabela 5-7: Contagem de partículas, para as 4 condições de

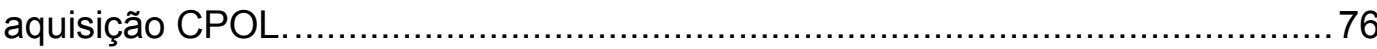

Tabela 8-1: Fração de área das hematitas (\%), para as 4 condições

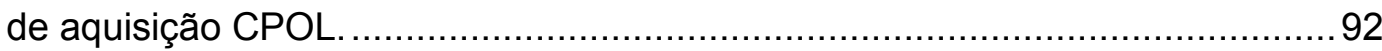

Tabela 8-2: Contagem de partículas, para as 4 condições de

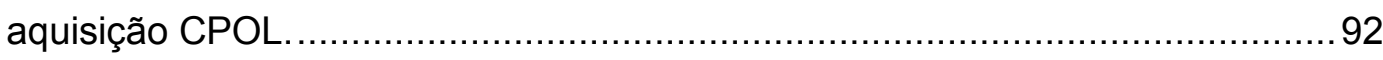

Tabela 8-3: Fração de área das hematitas (\%), para as 4 condições

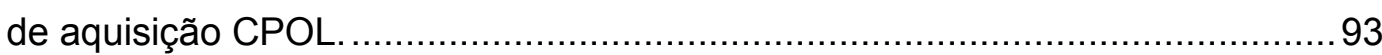

Tabela 8-4: Contagem de partículas, para as 4 condições de aquisição CPOL. 


\section{Lista de Abreviaturas}

ADI Análise Digital de Imagens

CC Campo Claro

CCD Charge Coupled Device

CPOL Campo Polarizado

CoA Compactas pelo Método Analítico

CoS Compactas pelo Método Sintético

DEQM Departamento de Engenharia Química e de Materiais

Gr Granular

ICMM Conselho Internacional de Mineração e Metais

La Lamelar

LBP Local Binary Patterns

LMD Laboratório de Microscopia Digital

Lo Lobular

LPOL Polarização Linear

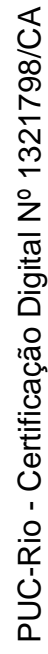

Ma Martita

Mc Microcristalina

MD Microscopia Digital

MLR Microscópio de Luz Refletida

NC Não compacta

NLM Non-Local Means

PADI Processamento e Análise Digital de Imagens

PDI Processamento Digital de Imagens

PIB Produto Interno Bruto

$\mathrm{ROI} \quad$ Region of Interest

SIFT Scale Invariant Feature Transform

Weka Waikato Environment for Knowledge Analysis 